9-1993

\title{
Photoabsorption Spectra of Atoms in Parallel Electric and Magnetic Fields
}

J. M. Mao

William \& Mary

K. A. Rapelje

William \& Mary

S. J. Blodgett-Ford

William \& Mary

John B. Delos

William \& Mary, jbdelos@wm.edu

A. König

See next page for additional authors

Follow this and additional works at: https://scholarworks.wm.edu/aspubs

Part of the Physics Commons

\section{Recommended Citation}

Mao, J. M.; Rapelje, K. A.; Blodgett-Ford, S. J.; Delos, John B.; König, A.; and Rinneberg, H., Photoabsorption Spectra of Atoms in Parallel Electric and Magnetic Fields (1993). Physical Review A, 48(3), 2117-2126.

https://doi.org/10.1103/PhysRevA.48.2117

This Article is brought to you for free and open access by the Arts and Sciences at W\&M ScholarWorks. It has been accepted for inclusion in Arts \& Sciences Articles by an authorized administrator of W\&M ScholarWorks. For more information, please contact scholarworks@wm.edu. 


\section{Authors}

J. M. Mao, K. A. Rapelje, S. J. Blodgett-Ford, John B. Delos, A. König, and H. Rinneberg 


\title{
Photoabsorption spectra of atoms in parallel electric and magnetic fields
}

\author{
J.-M. Mao,* K. A. Rapelje, S. J. Blodgett-Ford, and J. B. Delos \\ Department of Physics, College of William \& Mary, Williamsburg, Virginia 23187 \\ A. König ${ }^{\dagger}$ and H. Rinneberg ${ }^{\ddagger}$ \\ Institut für Atom- und Festkörperphysik, Freie Universität Berlin, 1000 Berlin 33, Germany
}

(Received 26 August 1992)

\begin{abstract}
Measurements have been made of the absorption spectrum of Ba from the $6 s 6 p^{1} P_{1}$ level to states near the ionization threshold in parallel electric and magnetic fields. The absorption spectrum shows oscillations superposed on a smooth background. Each oscillation is correlated with a closed orbit of the electron. At strong electric fields, trajectories are regular, and closed orbits form orderly patterns. For weak electric fields, trajectories are chaotic, and many more closed orbits are present. Many of these are produced by bifurcations as the electric field is reduced.
\end{abstract}

PACS number(s): 32.60. $+\mathrm{i}, 32.80 .-\mathrm{t}, 05.45 .+\mathrm{b}$

\section{INTRODUCTION}

The absorption spectrum of an atom in a magnetic field to states near the ionization threshold was first measured by Garton and Tomkins [1]. They found the absorption as a function of energy to be a set of oscillations superimposed on a smooth background. Later the group led by Welge [2] showed that the absorption consisted of multiple oscillations, and that each oscillation was correlated with a closed classical orbit of the electron moving under the combined Coulomb and Lorentz forces. A general theory describing this phenomenon was developed [3], which gives formulas for the magnitude and phase of each oscillation from properties of classical orbits and quantum wave functions for the electron.

In this paper we report measurements and calculations of the absorption spectrum in parallel electric and magnetic fields [4]. Again oscillations are visible, and each oscillation corresponds to a closed electron orbit. The closed-orbit theory accurately reproduces most of the experimental observations.

By measuring photoionization rate versus photon energy (excitation energy) at several field strengths, we are able to observe bifurcations of closed orbits of the electron. Mathematical theory has shown that bifurcations of periodic orbits occur in certain characteristic ways. For example, in a common type of "period-doubling" bifurcation, a stable periodic orbit becomes unstable, and a new stable orbit of twice the period is created. In fact, in Hamiltonian systems with two degrees of freedom, there are precisely five typical types of bifurcation $[5,6]$. This general theory needs modification to apply to the present case: our system has certain symmetries, and the experiments are sensitive to closure times rather than to periods of the orbits. With these modifications, the theory classifies and describes many bifurcations that are visible in the experimental data. We will show that pitchfork bifurcations, period doublings, four-island chains, and other bifurcations of electron orbits can be identified in the experimental data.

\section{EXPERIMENTS}

A well-collimated beam of $\mathrm{Ba}$ atoms in their ground state $6 s^{2}{ }^{1} S_{0}$ was intersected at right angles by two counterpropagating laser beams. The continuous wave (cw) dye lasers were operated with dyes rhodamine 110 and stilbene 3 to produce tunable laser radiation at $\lambda_{1} \approx 554$ $\mathrm{nm}$ and $\lambda_{2} \approx 417 \mathrm{~nm}$, respectively, stabilized to a bandwidth of about $1 \mathrm{MHz}$. One laser beam with a $\pi$ polarized electric field excited the $\mathrm{Ba}$ atoms to the $6 s 6 p^{1} P_{1}$ intermediate level. The second cw dye laser beam, also $\pi$ polarized, induced transitions to Rydberg states close to the zero-field ionization threshold $(-0.2$ $\mathrm{cm}^{-1} \leq E \leq 1.1 \mathrm{~cm}^{-1}$ ). Excitation was monitored by counting $\mathrm{Ba}^{+}$ions, converted from highly excited $\mathrm{Ba}$ atoms by collisional ionization, autoionization, or field ionization. For this purpose Ba ions were focused onto the entrance aperture of a quadrupole mass filter and detected by an electron multiplier. Spectra were recorded by keeping the first laser tuned to the resonance transition $6 s^{2}{ }^{1} S_{0} \rightarrow 6 s 6 p{ }^{1} P_{1}\left(\lambda_{1}=553.702 \mathrm{~nm}\right)$ and scanning the frequency of the second dye laser. A marker cavity allowed frequency separations to be measured with an accuracy better than $75 \mathrm{MHz}$.

The magnetic field, produced by an electromagnet and stabilized by a Hall probe, was oriented along the atomic beam in order to suppress motional Stark effects. The atomic beam was fed through a central bore in the yoke and pole pieces of the magnet (Fig. 1). The bore contained the quadrupole mass filter in the upper part and the barium oven in its lower part. The magnetic-field strength $(970 \mathrm{G})$ was inferred from measurements of the Zeeman splitting of the $6 s 6{ }^{1} P_{1}$ intermediate level. For this purpose the frequency of the first dye laser was scanned across the resonance transition and fluorescence emitted was imaged onto a photomultiplier tube followed by lock-in detection of the photomultiplier signal. In this way the magnetic-field strength was determined with a typical accuracy of $\pm 10 \mathrm{G}$.

External electric fields, also oriented parallel to the 


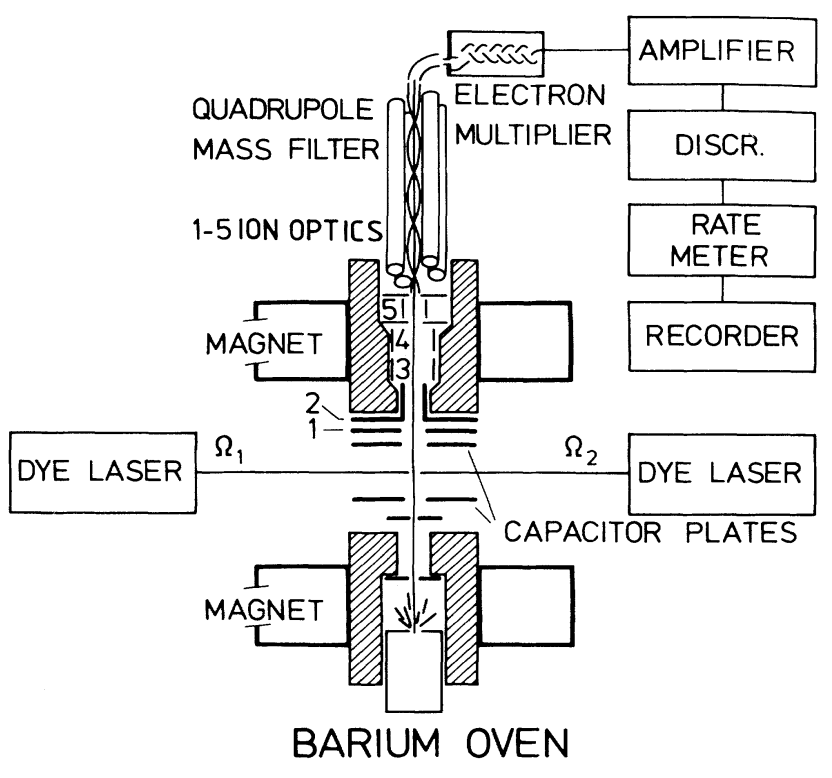

FIG. 1. Schematic diagram of the experimental apparatus.

atomic beam, were produced by a parallel-plate capacitor with central holes in the lower and upper plates for the atomic beam to pass through. Inside the interaction volume defined by the laser beam and the atomic beam, the electrical-field strength was smaller by about $3 \%$ compared to that calculated for an ideal parallel-plate capacitor because of the holes in the capacitor plates. Calibration of the electric-field strength was achieved by comparing at one field strength the observed location of peaks in the Fourier transform of the absorption spectrum with the location predicted by closed-orbit theory.

The $\mathrm{Ba}^{+}$count rate, being proportional to the photoabsorption cross section, shows modulations as a function of photon energy, superimposed upon a smooth background. Typical spectra obtained in parallel electric and magnetic fields are shown in Figs. 1(a)-1(f) of Ref. [4]. There, the origin of the frequency scale corresponds to the zero-field threshold and was deduced by measuring the wavelength of the second dye laser radiation. The energy resolution of the experimental spectra observed was about $0.4 \mathrm{GHz}$; it was limited by the time constant of the counting electronics (rather than by the bandwidth of the dye lasers or broadening of individual Rydberg levels due to stray electric fields and residual motional Stark effects). Some experimental results, shown as heavy curves in Fig. 2 of this paper, were calculated by Fourier transformation of autocorrelation functions of the experimental spectra [see also Figs. 1(g) $-1(1)$ of Ref. [4]]. Because of the energy resolution of the recorded spectra, the Fourier-transformed (power) spectra are limited to recurrence times $T / T_{c} \leq 5$ and are shown up to $T / T_{c}=3$ in Fig. 2. The large, distinct peaks establish that the fluctuations are superpositions of sinusoidal oscillations, having the form $C_{k} \sin \left(T_{k} E+\Delta_{k}^{0}\right)$, where $T_{k}$ is the time of a peak and $\left|C_{k}\right|^{2}$ is the height for that peak in the power spectrum.

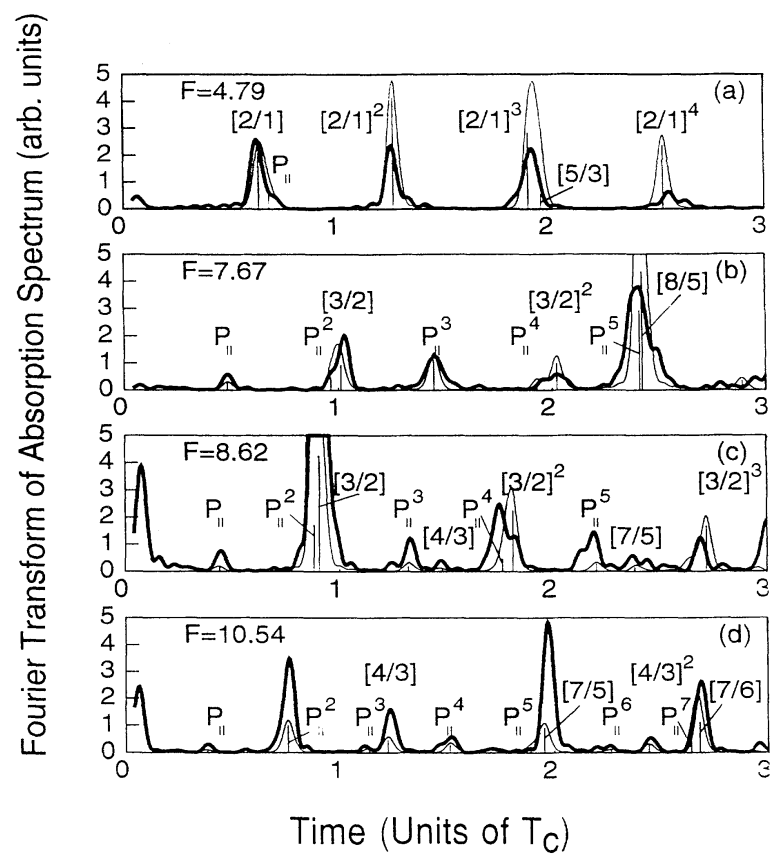

FIG. 2. The recurrence spectrum, i.e., the squared Fourier transform of the absorption spectrum. The thicker curves show the experimental results. The needles show the square of the absorption amplitude, i.e., $\left|C_{k n}\right|^{2}$, calculated by using Eqs. (3.9), (A7), or (A8). They are smoothed by averaging [Eq. (3.10a)] and finite-range Fourier transformation [Eq. (3.10b)] to obtain the thinner curves in the figure. The label near each needle is for the associated closed orbit. The shape of these closed orbits can be found in Figs. 3(a) and 3(b). The superscript of the labels is the number of repetitions of the closed orbit.

We call graphs like Fig. 2 "recurrence spectra," since (as explained below) each peak corresponds to an electron orbit that returns to the atom. $T_{k}$ is the return time and we call $\left|C_{k}\right|^{2}$ the "recurrence strength."

It is evident that the recurrences change significantly with changing electric field. The recurrences also change as the laser frequency and electron energy are varied. It is now known that scaled-variable experiments (varying photon energy and electric or magnetic field simultaneously) produce cleaner and better-resolved recurrence spectra [2]. This approach was not available to us when these measurements were made. Instead we used a range of photon energy sufficiently small that recurrence times would be nearly constant, but also sufficiently large that Fourier transformation would give narrow peaks.

\section{APPLICATION OF THE CLOSED-ORBIT THEORY}

In this section we will use the closed-orbit theory [3] to compute the modulations in the photoabsorption cross section for a hydrogen atom in parallel electric and magnetic fields. We will show that these calculations are generally consistent with the experimental measurements of the absorption spectrum of $\mathbf{B a}$. 


\section{A. System}

A hydrogen electron in a state with magnetic quantum number equal to zero is in a uniform electric field $\mathbf{F}$ and a uniform magnetic field $\mathbf{B}$. The fields are parallel and their common direction is chosen to be the positive $z$ axis. The Hamiltonian of this system is given by

$H=\frac{1}{2 m}\left(p_{\rho}^{2}+p_{z}^{2}\right)-\frac{e^{2}}{\left(\rho^{2}+z^{2}\right)^{1 / 2}}+\frac{1}{8} m \omega_{c}^{2} \rho^{2}+e F z$

where $m$ and $e$ are, respectively, the mass and charge of the electron, and $\omega_{c}=e B / m c$ is the cyclotron frequency. Here we used cylindrical coordinates $(\rho, z, \phi)$ and the $\phi$ motion has been separated. We define the scaling factor $\gamma=B / B_{0}$, where $B_{0}=m^{2} e^{3} c / \hbar^{3}=2.35 \times 10^{5} \mathrm{~T}$, and use the scaled variables

$$
\mathbf{r}=\frac{1}{2} \gamma^{2 / 3} \mathbf{r}, \quad \mathbf{p}=\gamma^{-1 / 3} \mathbf{p},
$$

where $\mathbf{r}=(\rho, z)$ and $\mathbf{p}=\left(p_{\rho}, p_{z}\right)$. The Hamiltonian becomes

$H=\frac{1}{2}\left[p_{\rho}^{2}+p_{z}^{2}-\frac{1}{\left(\rho^{2}+z^{2}\right)^{1 / 2}}+\rho^{2}+4 F \gamma^{-4 / 3} z\right]$.

Here we have used atomic units (i.e., $m=e=\hbar=1$ ). We further transform the coordinates to semiparabolic coordinates [7] $(u, v, \tau)$,

$$
z=u^{2}-v^{2}, \quad \rho=2 u v, \quad \frac{d \tau}{d t}=\frac{1}{4 r(t)},
$$

where $r=\left(\rho^{2}+z^{2}\right)^{1 / 2}$, and obtain the Hamiltonian

$$
\begin{aligned}
h= & \frac{1}{2}\left(p_{u}^{2}+p_{v}^{2}\right)-4\left(E \gamma^{-2 / 3}\right)\left(u^{2}+v^{2}\right)+8 u^{2} v^{2}\left(u^{2}+v^{2}\right) \\
& +8\left(F \gamma^{-4 / 3}\right)\left(u^{4}-v^{4}\right)=2 .
\end{aligned}
$$

This form of Hamiltonian will be used throughout the rest of this paper. This Hamiltonian is an even function of each coordinate and each momentum

$$
h=f\left(u^{2}, v^{2}, p_{u}^{2}, p_{v}^{2}\right) .
$$

This symmetry has an important consequence that we will use later [8]. Every orbit that starts from the origin at $t=0$ and returns to the origin at time $t=T_{\text {closure }}$ continues in a straight line through the origin and then moves on a path that brings it back again to the origin such that it is periodic with period $T_{\text {period }}=2 T_{\text {closure }}$. Conversely, every periodic orbit that goes through the origin consists of two closed orbits having closure time equal to half the period. The two closed orbits are related by inversion through the origin.

\section{B. Formulas for photoabsorption spectrum}

The photon-absorption rate is proportional to the oscillator-strength density $D f(E)$, where $E$ is the energy of the electron after photon absorption. $D f(E)$ has two terms,

$$
D f(E)=D f_{0}(E)+D f_{1}(E)
$$

Here $D f_{0}(E)$ is the smooth background term, equal to the oscillator-strength density that would be obtained in the absence of the external fields. $D f_{1}(E)$ is the oscillatory term arising from the closed orbits and their repetitions,

$$
D f_{1}(E)=\sum_{k} \sum_{n} C_{k n}(E) \sin \Delta_{k n}(E),
$$

where $k$ labels electron orbits that are closed at the nucleus, and $n$ labels the repetitions of each such closed orbit. The basic expressions for $C_{k n}$ and $\Delta_{k n}$ were given in Eq. (5.13) of Ref. [3]:

$$
\begin{aligned}
C_{k n}(E) e^{i \Delta_{k n}(E)}= & \left(E-E_{i}\right) 2^{19 / 4} \pi^{3 / 2} r_{b}^{-1 / 4} \\
& \times\left(\sin \theta_{i}^{k} \sin \theta_{f}^{2}\right)^{1 / 2} A_{k n} \\
& \times \exp \left[i\left[S_{k n}+2 \sqrt{8 r_{b}}-\mu_{k n} \frac{\pi}{2}\right]\right) \\
& \times \exp (-i 3 \pi / 4) \bar{Y}\left(\theta_{i}^{k}\right) \bar{Y}^{*}\left(\theta_{f}^{k}\right) .
\end{aligned}
$$

Here $S_{k n}+2\left(8 r_{b}\right)^{1 / 2}$ is the action around the closed orbit starting and ending at the nucleus, $A_{k n}$ is the classical amplitude associated with the closed orbit (the amplitude of the returning wave), and $\bar{Y}(\theta)$ is the angular distribution of the outgoing waves. Further details are given in Ref. [3].

In this paper we incorporate several recently developed refinement of the closed-orbit theory [8,9]. Recurrences associated with the orbit parallel to the fields are included, and a modified version of Eq. (3.9) is used that allows us to compute the recurrence strength for the $n$th return of an orbit from properties of the orbit on its first return (see Appendix).

With these refinements, our formulas describe the oscillations in the photoabsorption spectrum of hydrogen atoms in parallel fields. In the following section, we will use these formulas to calculate the Fourier transforms of the absorption spectrum, and compare the results with the experiment. To make the results analogous to the experiments on $\mathrm{Ba}$, we take the initial state of hydrogen to be the $2 p_{m=0}$ state. All calculations were made at $B=970 \mathrm{G}, E=0.6 \mathrm{~cm}^{-1}$ (the center of the range of measurements) at electric fields specified in the graphs.

\section{Comparison with experiments}

$D f_{1}(E)$ in Eq. (3.8) is a sum of sinusoidal oscillations, each associated with a closed orbit. Hence its Fourier transform is a set of $\delta$ functions, each representing a closed orbit, shown as the needles in Fig. 2. A needle is located at a time $T$ equal to the return time of the associated closed orbit, in units of the cyclotron period $T_{c}=2 \pi m c / e B$. The height of each needle is the recurrence strength $\left|C_{k n}\right|^{2}$ for that orbit. The ratio of integers near a needle is the label for the associated closed orbit whose picture is shown in Fig. 3.

Since experimental spectra were recorded with limited spectral resolution due to the time constant of the counting electronics, it is reasonable to average $D f_{1}(E)$ over the energy $E$ in a small range from $(E-\Delta E)$ to $(E+\Delta E)$ : 
$\overline{D f_{1}(E)}=\int_{E-\Delta E}^{E+\Delta E} D f_{1}\left(E^{\prime}\right) \frac{1}{\sqrt{2 \pi} \Delta E} \exp \left[-\frac{\left(E^{\prime}-E\right)^{2}}{2(\Delta E)^{2}}\right] d E^{\prime}$

We use the spectral range actually measured in the experiment (i.e., $E_{1}=-0.2 \mathrm{~cm}^{-1}$ and $E_{2}=1.4 \mathrm{~cm}^{-1}$ ) as the limits of the integral in the Fourier transformation, and define

$$
\widetilde{D} f_{1}(T)=\int_{E_{1}}^{E_{2}} \overline{D f_{1}(E)} e^{-i E T / \hbar} d E
$$

The square of this Fourier transform gives the fine curves around the needles in Fig. 2.

The experimental and theoretical results agree with each other quite well, as shown in Fig. 2. The most important discrepancy we find between theory and experiment is that the experimental peaks for the longer-time orbits are often smaller than the theoretical ones. This is reasonable, since longer orbits are more difficult to detect experimentally than shorter ones.

Why does a calculation on hydrogen agree with an experiment on barium? (i) The relevant closed orbits are very large, and the electron spends most of its time at distances around $10^{4} a_{0}$ from the atom. All it can see is a positive charge and the external fields. (ii) The initial state of $\mathrm{Ba} 6 s 6 p^{1} P_{1}$ has the shape of a $p$ wave, and spin- orbit coupling can be neglected. Dipole matrix elements connect this to outgoing $s$ waves and $d$ waves, and the angular distribution of the outgoing waves is described by the factor $\bar{Y}(\theta)$ in Eq. (3.9) or Eq. (A7). The outgoing waves are similar in barium and hydrogen, since the ratio of the dipole matrix elements connecting the $6 s 6 p^{1} P_{1}$ $m=0$ intermediate level with the $6 s(n+4) s{ }^{1} S_{0}$ and $6 s(n+3) d{ }^{1} D_{2} m=0$ Rydberg states is similar to that of the matrix elements between the states $2 p m=0$ and $n s$, $n d m=0$ in hydrogen. This can be inferred from zerofield spectra of the ${ }^{1} S_{0}$ and ${ }^{1} D_{2}$ Rydberg states of Ba and calculations using hydrogenic wave functions. (iii) The $\mathrm{Ba}^{+}$core produces a small phase shift to the outgoing waves [9]. However, the recurrence spectrum is not very sensitive to this.

This last point needs more discussion. Suppose we model the effect of the $\mathrm{Ba}^{+}$ion core as a short-range potential modifying the Coulomb potential at distances $\sim 1 a_{0}$. Suppose also we treat the electron motion semiclassically even at these small distances. The result is that the trajectories are substantially changed near the core. In a Coulomb field, orbits are sent back in the direction from which they came, but with the core, orbits at small impact parameter are widely scattered. As a result, the core itself produces chaotic trajectories.

In reality, however, the core affects only the phase shifts of the lowest one or two partial waves, and we be-

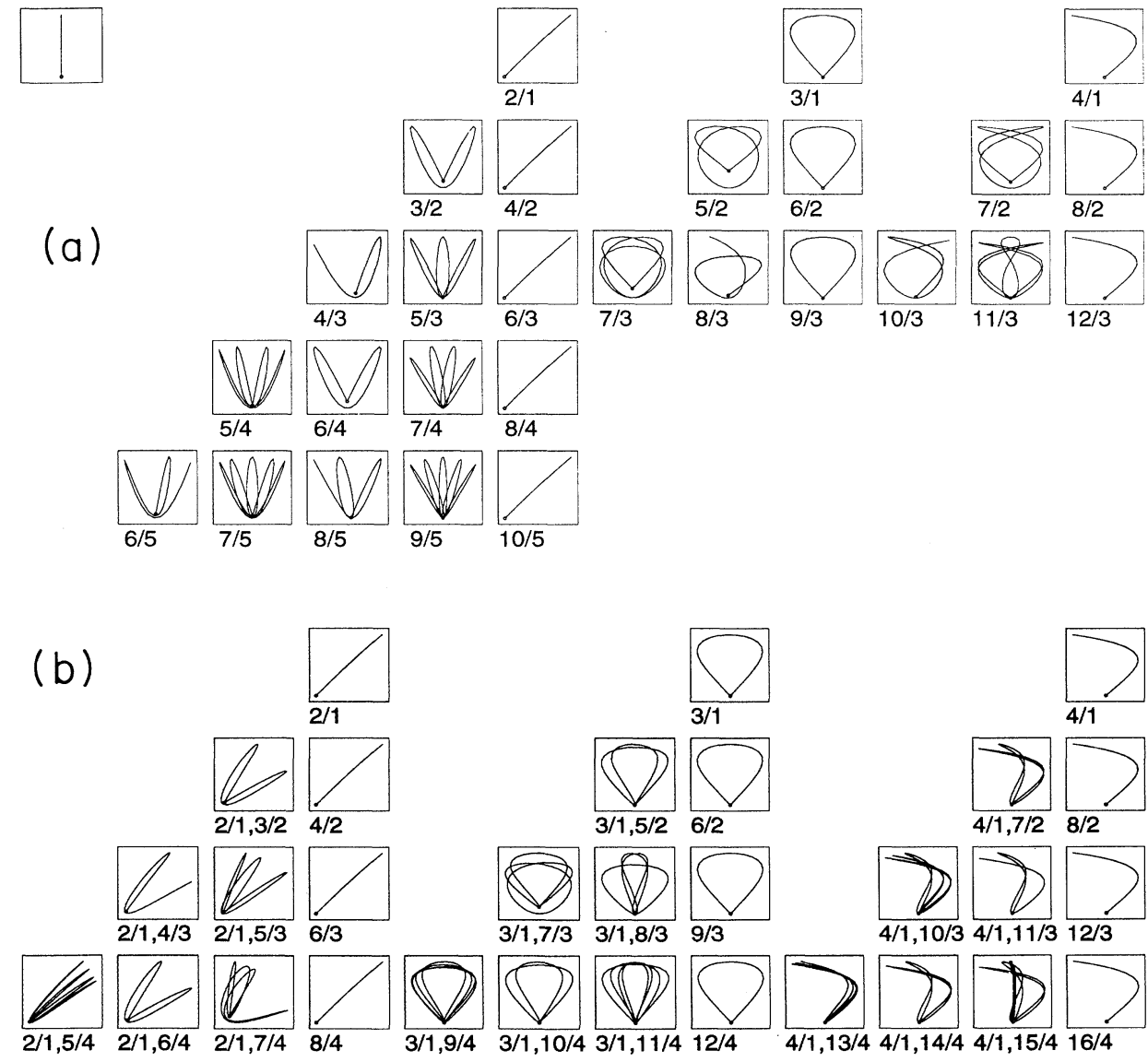

FIG. 3. Some closed orbits, arranged in terms of their parentage. (a) Closed orbits that bifurcte from the parallel orbit $\boldsymbol{P}_{\|}$. The orbits in the first row are created in the closure-1 bifurcation of $P_{\|}$, those in the second row in the closure- 2 bifurcations of $P_{\|}$, and so on. Each orbit is labeled by a ratio of integers $M / n$, explained in the text. (b) Closed orbits created in bifurcations of orbit [2/1], [3/1], and [4/1] shown in (a). Their labels have two ratios: the first labels the parent orbit and the second labels the offspring. 
lieve that a semiclassical treatment confuses the picture. In Ref. [9], we incorporated a quantum description of scattering by ion cores into the closed-orbit theory, and showed that the main effect of the core is a small change of phase of the oscillations in the absorption spectrum.

\section{BIFURCATIONS OF PERIODIC ORBITS}

\section{A. Introductory remarks}

The character of classical orbits of an atomic electron perturbed by a magnetic field is very different from that of an atomic electron perturbed by an electric field. Trajectories of an electron in a hydrogen atom in an electric field are all regular, and there is a full set of conservation laws (since the Hamilton-Jacobi equation is separable). In a magnetic field, however, at energies near threshold, the trajectories are highly chaotic: surface-of-section calculations show no visible order, and islands of stability are either tiny or nonexistent. This distinction between order and chaos has important consequences for the closed orbits of the system.

Recall that in $(u, v)$ space, each relevant closed orbit is half of a periodic orbit. In a regular system with two degrees of freedom, periodic orbits fall into orderly families. Periodic orbits in the electric-field case will be discussed in a future paper by Gao and Delos. At energies below the zero-field threshold, there is a stable orbit lying parallel to the electric field. It is surrounded by quasiperiodic orbits (tori), in which the frequency for $u$ oscillation (along the $z$ axis) is approximately constant, while the frequency for $v$ oscillation (across the $z$ axis) decreases with increasing amplitude of $v$ motion. Embedded in this continuous set of tori are rational tori. Each rational torus consists of a continuous one-parameter family of periodic orbits, all having the same frequency ratio $\omega_{v} / \omega_{u}=m / n$. Within each family is a single orbit that touches the nucleus. Only this orbit (and its time reverse) can produce a visible recurrence. It follows that closed orbits of any given period are isolated, and fall into orderly patterns.

Now let us raise the energy above threshold and turn on the magnetic field weakly. Raising the energy tends to destablilize the central orbit, but turning on the magnetic field stabilizes it again. It follows that the picture discussed above qualitatively describes the orbits of the electron at energies above threshold when electric effects dominate magnetic effects. Quantitative surfaces of section for this case were shown in Fig. 4 of Ref. [4].

Now let us reduce the electric field, keeping the magnetic field fixed. We know from earlier calculations that if $F=0$, surfaces of section show no visible order. In such systems, periodic orbits are isolated, unstable, and typically they do not fall into any global patterns [11].

Furthermore, chaotic systems have many more longperiod orbits than do orderly systems. This fact was established by Poincaré and Birkhoff (a simple form of their argument is given by Jackson [12]). In a regular system with two degrees of freedom the number of periodic orbits of given energy having period less than $T_{\max }$ is proportional to $T_{\max }^{2}$, whereas in a chaotic system the number is proportional to $\exp \left(a T_{\max }\right) / T_{\max }$, where $a$ is a con- stant. It follows that as order goes over to chaos, periodic orbits proliferate.

How does the system go from a small, orderly set of periodic orbits to a vast incomprehensive profusion of them? As chaos develops, new periodic orbits bifurcate out of existing ones, and also new orbits are created "out of nowhere."

This phenomenon becomes visible in our system when we reduce the electric field holding the magnetic field fixed. It is somewhat masked by two other facts: (i) we can only see orbits having short periods, and (ii) the periods of the important orbits increase with decreasing $F$. Nevertheless the phenomenon is visible in the experimental measurements, and perfectly plain in the calculations.

\section{B. Essential results from bifurcation theory}

As mentioned earlier, the essential aspects of bifurcation theory were developed by Meyer and further developed (for systems with symmetries) and applied to the diamagnetic Kepler problem in Ref. [6]. The essential results are the following.

(i) Given a periodic orbit which exists at some value of electric field $F$, as we vary the electric field, the orbit generally changes its shape smoothly. It may be created or destroyed only at special, isolated values of $F$.

(ii) An unstable periodic orbit cannot bifurcate. It can be destroyed only by collision with a stable orbit.

(iii) Stable periodic orbits can bifurcate to create new orbits of longer period in the following way. If an orbit is stable, then in general its neighbors oscillate about it quasiperiodically. However, at certain values of the parameter $F$, they oscillate periodically and their period is some multiple $n$ of the period of the stable orbit. At such values of $F$, new periodic orbits can be created, and their period is $n$ times that of the original stable orbit,

$$
T^{\text {new }}=n T_{\text {stable }} \text {. }
$$

(iv) Most commonly, a stable and an unstable pair of orbits of period $n T_{\text {stable }}$ are created. On a surface of section, they form an $n$-island chain, and we call this an island-chain bifurcation. Exceptional cases occur if $n=4,3,2$, or 1 .

The above statements describe typical bifurcations of periodic orbits. The theory we need differs in several ways, as stated earlier: (i) the experiments are sensitive to closure times, not periods; (ii) the experiments detect only those orbits that are closed at the nucleus; (iii) the system has a number of symmetries which lead to nongeneric bifurcations.

Details of the theory are given in Refs. [6] and [8]. The one quantitative formula we need tells the location of bifurcations from properties of the Poincare half-map (see Appendix). The quantity $T_{1}^{\prime}$ defined in Eq. (A3) can be calculated for each closed orbit as a function of electricfield strength. When $T_{1}^{\prime}(F)$ passes through certain values, then new orbits that produce recurrences may be created. The closure time of the newly created orbit is related to the closure time of the parent orbit by the follow- 
ing prescription:

$$
T_{1}^{\prime}=\left\{\begin{array}{l} 
\pm 2 \text { same closure time } \\
0 \text { closure time doubled } \\
\pm 1 \text { closure time tripled } \\
\pm \sqrt{2} \text { closure time quadrupled }
\end{array}\right.
$$

The general formula is $T_{1}^{\prime} \equiv \pm 2 \cos (\pi m / n)$, and the closure time is multiplied by $n$ [13]. Equivalent information comes from the angle $\alpha_{1}^{\prime}$, also defined in Eq. (A3). This angle represents the rate at which the neighbors of a given closed orbit wind around it in phase space. Bifurcation points occur when $\alpha_{1}^{\prime}$ is a rotational fraction of $\pi$,

$$
\alpha_{1}^{\prime}=\frac{m}{n} \pi
$$

Newly created orbits are labeled by a fraction $M / n ; M$ is chosen to be a certain Maslov index associated with the bifurcation.

\section{Bifurcations of closed orbits}

If the electric-field strength $F$ is sufficiently large, we find that there is only one closed orbit, lying parallel to the electric field, and we find that it is unstable. If the electron is launched from the nucleus at any nonzero angle from the $z$ axis, it is pulled downward by the strong electric field, and it never returns. (For $B=970 \mathrm{G}$, $E=0.6 \mathrm{~cm}^{-1}$, this happens for $F \geq 54 \mathrm{~V} / \mathrm{cm}$.) When $F$ decreases, other closed orbits bifurcate from this parallel orbit, then other closed orbits bifurcate from those.

The values of $F$ at which bifurcations occur is predicted by computing $T_{1}^{\prime}$ as a function of $F$. The result for the parallel orbit is shown in Fig. 4. As stated above, when $T_{1}^{\prime}= \pm 2$ a new closed orbit having the same closure time as the parallel orbit might be created. In fact, at half of these points the newly created periodic orbit of the map is not closed at the nucleus. At the points marked by dots, a new orbit closed at the nucleus is created. Each is labeled $[M / 1]$, and is drawn in Fig. 3(a).

These bifurcations can be categorized as "pitchfork bifurcations." Since $T_{1}^{\prime}$ passes right through \pm 2 , the parallel orbit goes from stable to unstable, and in fact two new stable orbits are created in $(u, v)$ space, which are reflections of each other through the $u$ axis. (When $T_{1}^{\prime}=+2$, the period of the new orbit, as it is seen in the Poincaré half-map, is the same as the period of the parallel orbit, and the bifurcation is an ordinary pitchfork. When $T_{1}^{\prime}=-2$, the period of the new orbit as seen in the half-map is twice the period of the parallel orbit, and the bifurcation is a period doubling. Since the experiments are sensitive to closure times rather than to periods, this distinction is not important to us.)

At field strengths $F$ such that $\alpha_{1}^{\prime}=m \pi / 2$, or $T_{1}^{\prime}=0$, an orbit with twice the closure time of the parallel orbit may be created. The first such point occurs just below 10 $\mathrm{V} / \mathrm{cm}$, and it creates the [3/2] orbit in Fig. 3(a). The next such point occurs when $\alpha_{1}^{\prime}=\pi$, and the "newly created closure-2" orbit is actually the repetition of the [2/1] or-

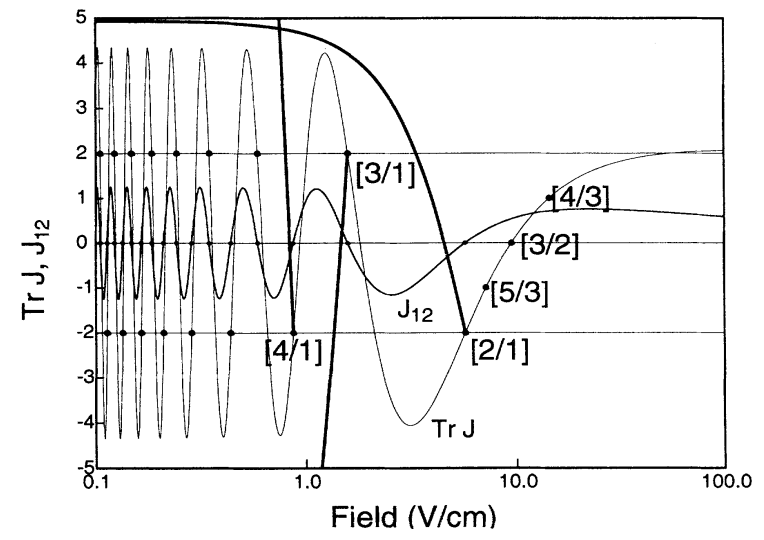

FIG. 4. Some matrix elements of $J$ evaluated always at the first return of orbits. Fine curve: $\operatorname{Tr} \underline{J}$ for the parallel orbit. When it passes \pm 2 the orbit undergoes pitchfork or perioddoubling bifurcations. Medium curve: $J_{12}$ for the parallel orbit. It vanishes at those bifurcations, producing singularities in the classical amplitude. Heavy curve: $\operatorname{Tr} \underline{J}$ for the [2/1], [3/1], and [4/1] orbits. These also undergo bifurcations at corresponding values of this trace.

bit (and we call it $[4 / 2]$ or $[2 / 1]^{2}$ ). At $F \approx 1.8 \mathrm{~V} / \mathrm{cm}$, where $T_{1}^{\prime}$ passes through zero again, the orbit labeled $[5 / 2]$ is created, and so on.

The creation of the [3/2] orbit might be called a "closure-time-doubling" bifurcation. It is similar to the famous period-doubling bifurcations only in that the relevant time for the new orbit is twice the time of the old orbit. However, it has quite a different structure from the usual period doubling (a stable orbit goes unstable, and a new orbit of period 2 appears). This closure-timedoubling bifurcation has the structure of a periodquadrupling (4-island-chain) bifurcation of the Poincare half-map, and the original parallel orbits stays stable before and after the bifurcation.

Similarly all other orbits $[M / n]$ in Fig. 3(a) are created by closure-time multiplication of the parallel orbit. We call these "first-generation" orbits.

It happens that all the first-generation orbits are born stable. As $F$ decreases, they themselves bifurcate, and produce "second-generation" orbits. Examples are shown in Fig. 3(b). Each grandchild of the parallel orbit is labeled by its parent's name $[M / n]$ and by its own name $\left[M^{\prime} / n^{\prime}\right]$.

\section{Bifurcation diagram in (field, closure-time) space}

The bifurcations of closed orbits discussed in the previous section can also be illustrated in $F$ versus $T$ space where $F$ is the electric-field strength and $T$ is the closure time of the orbit (Fig. 5).

The leftmost curve in Fig. 5 with label $P_{\|}$represents $T$ versus $F$ for the parallel orbit. As $F$ becomes smaller, the electron goes further from the nucleus, and therefore the period is longer. This curve has its first branch at $F=5.66 \ldots \mathrm{V} / \mathrm{cm}$ when orbit $[2 / 1]$ is created. This 


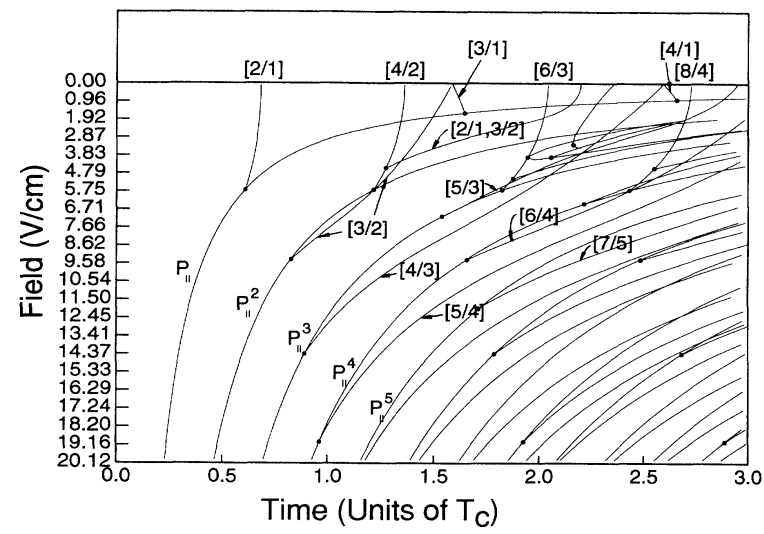

FIG. 5. Bifurcations of closed orbits shown as return-time versus electric-field strength. Each continuous smooth curve is the return time $T$ of a specific closed orbit, as a function of the electric-field strength $F$. It can be seen that orbits $[M / n]$ are created in the closure- $n$ bifurcations of the parallel orbit, and that they themselves bifurcate further.

branching is the closure-1 (pitchfork) bifurcation discussed before. The new orbit has its own curve for $T$ as a function of $F$. Similarly, we can see the bifurcations which create orbits [3/1] and [4/1] branching out from the same curve.

The second curve from the left in the lower part of the picture, labeled $P_{\|}^{2}$, is for the second repetition of the parallel orbit. Again, this curve branches at bifurcations, where orbits of twice the closure time of the parallel orbit are created. Only orbits $[3 / 2]$ and $[4 / 2] \equiv[2 / 1]^{2}$ are plotted in the figure.

In higher-closure bifurcations of the parallel orbit, more orbits $[M / n], n=3,4, \ldots$, are created, and all these orbits bifurcate further to produce the second generation. Therefore we observe more and more branching in the right half of the figure.

Let us draw a horizontal line at $F=4.79 \mathrm{~V} / \mathrm{cm}$ in Fig. 5. It intersects the curves for orbit $[2 / 1], P_{\|},[2 / 1],{ }^{2} P_{\|}^{2}$, $[2 / 1],{ }^{3} P_{\|}^{3},[5 / 3], P_{\|}^{4}, \ldots$ sequentially. These intersections indicate the locations of peaks in the Fourier transforms of the photoabsorption spectra shown in Fig. 2.

\section{E. Fourier transforms of spectra in an overlay form}

The Fourier transforms of photoabsorption spectra given by the experiments, as shown in Fig. 2, are for selected values of the electric-field strength $F$. We can put these figures together with corresponding figures obtained at other field strengths in an overlay form as shown in Fig. 6. The heavy curve in Fig. 2(a) (experimental result for $F=4.79 \mathrm{~V} / \mathrm{cm}$ ), for instance, is now located above the horizontal line for $F=4.79 \mathrm{~V} / \mathrm{cm}$ in Fig. 6. This overlay plot then corresponds to a three-dimensional plot for the recurrence strength as a function of the field strength $F$ and the period of closed orbit $T$. The experiments have been done for $F=(0.958 \mathrm{~V} / \mathrm{cm}) N$, where $N=0,1,2, \ldots, 20$, except $N=13,16,17,18,19$.

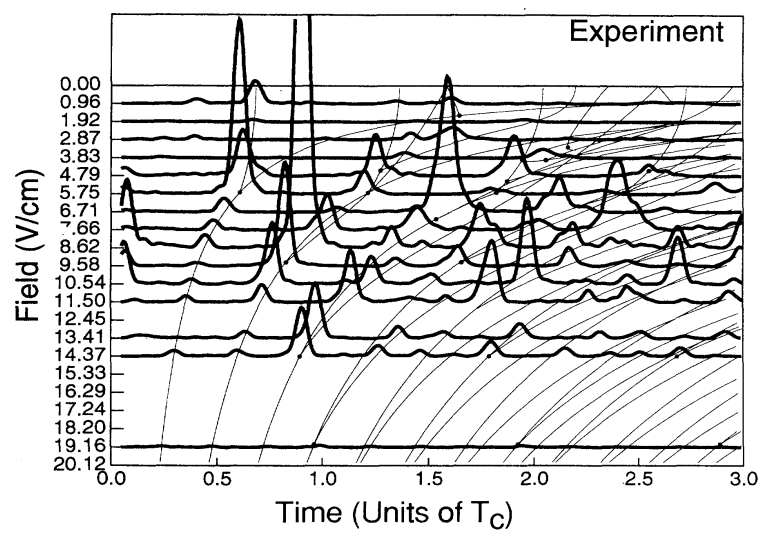

FIG. 6. Experimental overlay plot of the squared Fourier transforms of absorption spectrum at different $F$ values. The horizontal axis is the return time of closed orbit $T$ in units of the cyclotron period. The vertical axis is the electric-field strength $F$. The peaks may be considered to extend in a direction perpendicular to this $T F$ plane.

It can be seen in Fig. 6 that the recurrence peaks are located along the fine curves in the $F-T$ space, which are a duplication of Fig. 5, the predictions of the closed-orbit theory, and bifurcation theory. The predictions agree with experiments very well.

Similarly, we put the theoretical Fourier transform of photoabsorption spectrum for different $F$ values together in one figure, Fig. 7. Again the fine curves as a copy of Fig. 5. We have calculated, using Eq. (3.16), the Fourier transforms of spectra for $F=(0.958 \mathrm{~V} / \mathrm{cm}) N$, where $N=0,1,2, \ldots, 20$.

Comparing Figs. 6 and 7, the correspondence between theory and experiment is again pleasing. In particular, bifurcations are visible in two ways. At an $[m / n]$ bifurcation of any orbit, the spectral oscillation amplitude for the $n$th return of the orbit becomes very large. Also as

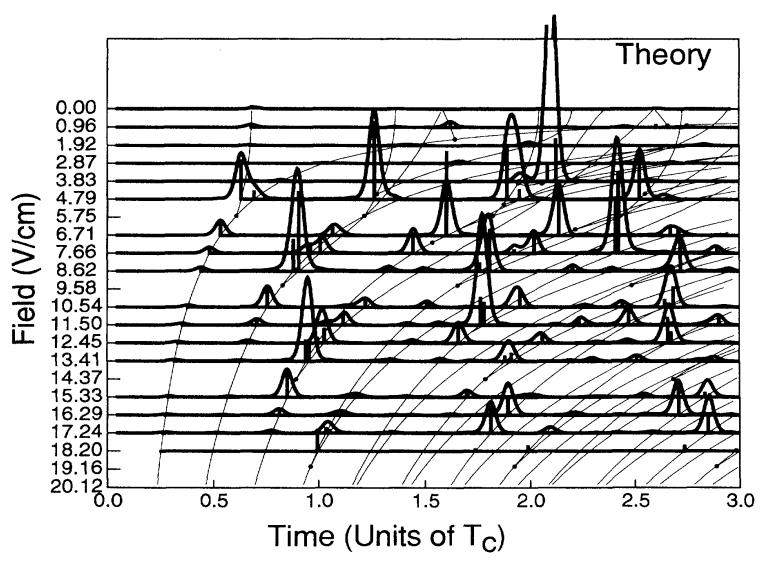

FIG. 7. Same as Fig. 6, but for theoretical results. The height of peak is the absolute square of $C_{k n}$ in Eq. (3.8) and is calculated according to Eq. (A7). 


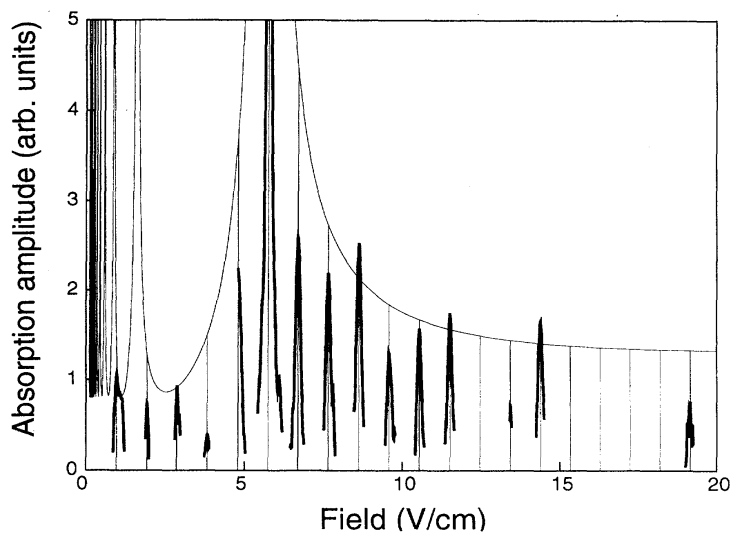

FIG. 8. The field dependence of the absorption amplitude for the parallel orbit.

we go through the bifurcation with $F$ decreasing, a single peak splits into two. Both of these phenomena are quite plain at many of the bifurcation points, marked by dots.

Actually, at a bifurcation the semiclassical theory used here has a problem. It does indeed predict that the spectral-oscillation amplitude will become very large - in fact it predicts an infinite amplitude. The physical reason for the singularity is connected with the argument surrounding Eq. (4.1). At the bifurcation, the neighbors of a periodic orbit oscillate about it with a period that is rationally related to the period of the orbit. After precisely $n$ repetitions the neighbors all come back together and are focused at the nucleus. Semiclassical theories predict an infinite amplitude at a focus, and they have to be repaired.

Near points of bifurcation we obtained peaks that were too large to draw in Fig. 8, so we omitted theoretical curves at $F=0.958 N$ with $N=6,10,15$, and 20. We see the same phenomenon in a different way by examining a single spectral-oscillation amplitude $C_{k n}$ as a function of electric-field strength. In particular, for the first closure of the parallel orbit, the amplitude $C_{\|, 1}$ is proportional to $\left|J_{12}\right|^{-1}$, and $J_{12}$ passes through zero at each $M$ bifurcation. We show this behavior in Fig. 8. The needles are the computed values of $\left|C_{\|, 1}\right|^{2}$ at various field strengths, and the envelope curve is proportional to $\left|J_{12}(F)\right|^{-2}$. The singularities are quite evident.

The heavier curves underneath the envelope are peaks corresponding to the parallel orbit extracted from the experimental data. The qualitative and even semiquantitative similarity between the heights of these peaks and the envelope of the needles is better than one might have expected.

\section{CONCLUSION}

Measurements of the absorption spectrum of atoms in parallel electric and magnetic field give information about closed classical orbits of the active electron. Each closed orbit produces an oscillation in the absorption as a function of energy, and a peak in the Fourier transform of the absorption. As the electric field is reduced, the character of the dynamics changes from orderly to chaotic, and closed orbits bifurcate and proliferate. At a bifurcation, the observed peaks in the Fourier-transformed absorption become very large, then split into two. Bifurcation theory for Hamiltonian systems describes the types of bifurcations that typically occur. Comparing calculations to experiments, pitchfork, period-doubling, 4- and 6-island chains, and other bifurcations can be identified.

\section{ACKNOWLEDGMENTS}

This research was supported by the Jeffress Foundation, the Office of Naval Research and National Science Foundation, through grants to the College of William and Mary and to the Institute for Theoretical Atomic and Molecular Physics at the Harvard-Smithsonian Center for Astrophysics. J.B.D. specially thanks the members of the Institute for their hospitality.

\section{APPENDIX: REFINEMENTS OF CLOSED-ORBIT THEORY}

(i) The classical amplitude on any return of any closed orbit is related to an element of the reduced monodromy matrix associated with that return. In regularized variables, let a closed orbit start at $\left(u, v, p_{u}, p_{v}\right)=\left(0,0, p_{u}^{0}, p_{v}^{0}\right)$ and let its neighbors start at $\left(0, d v, p_{u}^{0}+d p_{u}, p_{v}^{0}+d p_{v}\right)$. $d p_{u}$ is determined from $d v$ and $d p_{v}$ by the condition that the closed orbit and its neighbors have the same energy. We integrate Hamiltonian's equations of motion until $u(t)$ again passes through zero with either sign of $p_{u}$, and we record the new values of $v(t)$ and $p_{v}(t)$ as $v_{1}$ and $p_{v 1}$. The reduced monodromy matrix $\underline{J}(1)$ is defined as

$$
\underline{J}(1)=\left[\begin{array}{ll}
\frac{\partial v_{1}}{\partial v} & \frac{\partial v_{1}}{\partial p_{v}} \\
\frac{\partial p_{v 1}}{\partial v} & \frac{\partial p_{v 1}}{\partial p_{v}}
\end{array}\right] \equiv\left[\begin{array}{ll}
J_{11}(1) & J_{12}(1) \\
J_{21}(1) & J_{22}(1)
\end{array}\right]
$$

The argument (1) refers to the first return. The matrix on the $n$th return $J(n)$ is defined similarly. In Ref. [8] we have shown that for any return $n$ of any closed orbit (where the label $k$ is temporarily suppressed)

$$
A_{n}=\left|\frac{\dot{R}_{b}}{R_{b}} \cos \Theta_{i} \cos \Theta_{f}(n) J_{12}(n)\right|^{-1 / 2} \text {. }
$$

(A similar relationship was also used in Ref. [10].) Here $R_{b}$ is the boundary radius, $\dot{R}_{b}$ is the speed at this radius, and $\Theta_{f}(n)$ is the angle from which the electron approaches the atom on the $n$th return.

The calculation of $\left(v_{1}, p_{v 1}\right)$ from $\left(v_{0}, p_{v 0}\right)$ defined above, stopping every time that $u=0$ regardless of the sign of $p_{u}$, defines the Poincaré "half-map" (usually one stops only when $p_{u}>0$, but the symmetries make the half-map more convenient and appropriate).

(ii) The element of the reduced monodromy matrix on the $n$th return is related to properties of the reduced monodromy matrix on the first return [8]. Define $\alpha_{1}^{\prime}, T_{1}^{\prime}$, and $T_{n}^{\prime}$ such that 
$\cos \alpha_{1}^{\prime}=\operatorname{sgn}\left(J_{11}+J_{22}\right) \sqrt{J_{11} J_{22}}, \quad 0<J_{11} J_{22}<1$,

$\cos \left(2 \alpha_{1}^{\prime}\right)=\operatorname{sgn}\left(J_{11} J_{22}\right)\left(2 J_{11} J_{22}-1\right)$, otherwise ,

$T_{1}^{\prime}=\operatorname{sgn}\left(J_{11} J_{22}\right) 2 \cos \alpha_{1}^{\prime}$,

$T_{n}^{\prime}=\operatorname{sgn}\left(J_{11} J_{22}\right) 2 \cos \left(n \alpha_{1}^{\prime}\right)$,

where $\alpha_{1}^{\prime}$ is real with $0<\alpha_{1}^{\prime}<\pi$ if $0<J_{11} J_{22}<1$, and $\alpha_{1}^{\prime}$ is purely imaginary otherwise [and $\left.\cos \left(n \alpha_{1}^{\prime}\right)=\cosh \left(n\left|\alpha_{1}^{\prime}\right|\right)\right]$. Then

$$
\left|J_{12}(n)\right|=\left|J_{12}(1)\right|\left(\left|\frac{T_{n}^{\prime 2}-4}{T_{1}^{\prime 2}-4}\right|\right)^{1 / 2}\left\{\begin{array}{l}
1, n \text { odd , } \\
\left.|| \frac{J_{22}}{J_{11}} \mid\right)^{1 / 2},
\end{array}\right.
$$

$n$ even .

(A4)

In these formulas, all $J_{i j}$ mean $J_{i j}(1)$ unless otherwise indicated.

(iii) The Maslov index on the $n$th return is related to the Maslov index and the reduced monodromy matrix associated with the first return [8],

$$
\mu_{n}=n \mu_{1}+v_{n}
$$

where

$$
v_{n}=\left\{\begin{array}{ll}
\operatorname{Int} \frac{n \alpha_{1}^{\prime}}{\pi}, & J_{12}>0 \\
\operatorname{Int} \frac{n\left(\pi-\alpha_{1}^{\prime}\right)}{\pi}, & J_{12}<0 \\
0, & J_{12} J_{22}>0 \\
n-1, & J_{12} J_{22}<0
\end{array}\right\} \quad J_{11} J_{22}>1
$$

Again all $J_{i j}$ mean $J_{i j}(1)$ and "Int" means "the integer part of."

(iv) Oscillations related to the orbit that goes parallel to the field are included. In Ref. [3], this orbit was not included. If Eq. (3.9) were used for that orbit, we would find that the modulations in the spectrum associated with that orbit have zero amplitude [because of the $\left(\sin \theta_{i} \sin \theta_{f}\right)^{1 / 2}$ factor]. In fact, Eq. (3.9) does not apply to that orbit. An appropriate formula was developed in Ref. [9] for the zero-degree orbit.

With the refinements stated above, Eq. (3.8) becomes

$D f_{1}(E)=\left.D f_{1}(E)\right|_{\|}+2^{5} \pi^{3 / 2} \frac{m e}{\hbar^{2}}\left(E-E_{i}\right)\left(\frac{B}{B_{0}}\right)^{1 / 6} \sum_{\mathrm{CO}} \sin \frac{\theta_{i}}{2} \frac{1}{\sqrt{\left|J_{12}\right|}} \bar{Y}\left(\theta_{i}\right) \bar{Y}^{*}\left(\theta_{f}\right)$

$$
\begin{aligned}
& \left.\left.\times\left[|| \frac{\sin \theta_{f} / 2}{\sin \theta_{i} / 2} \mid\right]^{1 / 2} \sum_{n \text { odd }}+\left[\left|\frac{J_{11}}{J_{22}}\right|\right]^{1 / 4} \sum_{n \text { even }}\right]|| \frac{\sin \alpha_{1}^{\prime}}{\sin \left(n \alpha_{1}^{\prime}\right)} \mid\right)^{1 / 2} \\
& \times \sin \left[n\left[\frac{S_{1}}{\hbar}-\mu_{1} \frac{\pi}{2}-\pi\right]-v_{n} \frac{\pi}{2}+\frac{\pi}{4}\right],
\end{aligned}
$$

where $\theta_{i}$ is defined by $p_{v}^{\text {initial }}=2 \sin \left(\theta_{i} / 2\right), 0 \leq \theta_{i} \leq \pi ; \theta_{f}$ is defined likewise. Here $\left.D f_{1}(E)\right|_{\|}$is the contribution of the parallel orbit to the absorption spectrum

$$
\begin{aligned}
\left.D f_{1}(E)\right|_{\|}= & \left.2^{3} \pi \frac{m e}{\hbar^{2}}\left(E-E_{i}\right) \mid \frac{B}{B_{0}}\right)^{1 / 3} \frac{1}{\left|J_{12}\right|}|Y(0)|^{2} \\
& \times \sum_{n=1}^{\infty}\left|\frac{\sin \alpha_{1}^{\prime}}{\sin \left(n \alpha_{1}^{\prime}\right)}\right| \\
& \times \sin \left[n\left[\frac{S_{1}}{\hbar}-\mu_{1} \frac{\pi}{2}-\frac{\pi}{2}\right]-v_{n} \frac{\pi}{2}\right] .
\end{aligned}
$$

The first summation in Eq. (A7), $\Sigma_{\mathrm{CO}}$, is over all closed orbits other than the parallel orbit. The other sums in the square bracket are over all repetitions of each closed orbit, with $n$ the number of repetition. For those closed orbits that have $\theta_{i}=\theta_{f}$ or

$$
\begin{aligned}
& J_{11}=J_{22}, \\
& {\left[\left|\frac{\sin \theta_{f} / 2}{\sin \theta_{i} / 2}\right|\right)^{1 / 2} \sum_{n \text { odd }}+\left\lfloor\left|\frac{J_{11}}{J_{22}}\right|\right)^{1 / 4} \sum_{n \text { even }}=\sum_{n=1}^{\infty} .}
\end{aligned}
$$

(v) The monodromy matrix on the first return tells the location and character of bifurcations. Given an orbit which is closed at the nucleus, suppose we determine the monodromy matrix (A1) for perturbations of that orbit, and the quantity $\alpha_{1}^{\prime}$ defined in Eq. (A3). That orbit can bifurcate at values of $F$ such that

$$
\alpha_{1}^{\prime}=\frac{m}{n} \pi
$$

An equivalent expression is more useful in computations. The orbit is stable if

$$
\operatorname{sgn}\left(J_{11} J_{22}\right)>0,
$$

and in that case

$$
T_{1}^{\prime} \equiv 2 \operatorname{sgn}\left(J_{11}+J_{22}\right)\left(J_{11} J_{22}\right)^{1 / 2} .
$$

(If $J_{11}=J_{22}$, which is true for most of our orbits, $T_{1}^{\prime}$ is the 
trace of the Jacobian matrix.) The orbit can bifurcate if

$$
T_{1}^{\prime}= \pm 2 \cos (\pi m / n) \text {. }
$$

The bifurcation may or may not produce new orbits which are closed at the nucleus. If it does, then the first closure time of the new orbits is $n \times$ (the closure time) of the original orbit [13].

Our convention on $\alpha_{1}^{\prime}$ is $0 \leq \alpha_{1}^{\prime}<\pi$, so $m<n$. [This convention is required for (A6) to be valid.] To label the orbits, we found it convenient to use a fraction $[M / n]$ where $M$ is an integer closely related to $m$, defined as follows. We define the "two-dimensional Maslov index" $\mu$ of an orbit to be the Maslov index of the returning wave of that orbit, excluding those increments that arise from crossings of the $z$ axis or from touching the origin. We let the label $M$ of a newly bifurcated orbit be equal to $\mu$, unless the orbit bifurcates directly out of the parallel orbit, in which case $M=\mu+1$. It follows that at the bifurcation

$$
\cos \alpha_{1}^{\prime}=\cos (\pi m / n)= \pm \cos (\pi M / n) .
$$

*Present address: Department of Mathematics, Hong Kong University of Science and Technology, Clear Water Bay, Hong Kong.

†Present address: Daimler-Benz Forschungsinstitut Frankfort/M, Goldsteinstrasse 235, 6000 Frankfort/Main, Germany.

$\ddagger$ Present address: Physikalish-Technisch Bundesanstalt Berlin-Charlottenburg, Abbestrasse 2-12, 1000 Berlin 10, Germany.

[1] W. R. S Garton and F. S. Tomkins, Astrophys. J. 158, 839 (1969); K. T. Lu, F. S. Tomkins, and W. R. S. Garton, Proc. R. Soc. London Ser. A 362, 421 (1978); A. R. Edmonds, J. Phys. (Paris) Colloq. 31, C4-71 (1970).

[2] A. Holle, G. Wiebusch, J. Main, B. Hager, H. Rottke, and K. H. Welge, Phys. Rev. Lett. 56, 2594 (1986); J. Main, G. Wiebusch, A. Holle, and K. H. Welge, ibid. 57, 2789 (1986).

[3] M. L. Du and J. B. Delos, Phys. Rev. Lett. 58, 1731 (1987); Phys. Rev. A 38, 1896 (1988); 38, 1913 (1988).

[4] A preliminary account appeared in A. Konig, J. Neukammer, K. Vietzke, M. Mohl, H.-J. Grabka, H. Hieronymus, and H. Rinneberg, Phys. Rev. A 38, 547 (1988).
[5] K. R. Meyer, Trans. Am. Math. Soc. 149, 95 (1970); see also M. A. M. deAguiar, C. P. Malta, M. Baranger, and K. T. R. Davies, Ann. Phys. (N.Y.) 180, 167 (1987).

[6] J. M. Mao and J. B. Delos, Phys. Rev. A 45, 1746 (1992).

[7] E. L. Stiefel and G. Scheifele, Linear and Regular Celestial Mechanics (Springer-Verlag, New York, 1971).

[8] J. M. Mao and J. B. Delos, J. Stat. Phys. 68, 97 (1992).

[9] J. Gao and J. B. Delos, Phys. Rev. A 46, 1455 (1991).

[10] E. B. Bogomolnyi, Zh. Eksp. Teor. Fiz. 96, 487 (1989) [Sov. Phys. JETP 69, 275 (1989)].

[11] A partially successful scheme for labeling all orbits using simple rules was proposed, but it was later found not to be a complete one-to-one system.

[12] E. A. Jackson, Perspectives of Nonlinear Dynamics (Cambridge University Press, Cambridge, England, 1989), Vol. 2.

[13] This statement presumes that the fraction $m / n$ is expressed in lowest terms. Caution: In Ref. [8] we wrote in Eq. (3.5) a formula implying $\alpha_{1}^{\prime}=2 \pi j / m$, where here we have $\alpha_{1}^{\prime}=\pi m / n$. It is the same $\alpha_{1}^{\prime}$, but a different rational representation. The relationship between $(j, m)$ in Ref. [8] and $(m, n)$ here is nontrivial. 